\section{WEB WATCH}

Gene stories

- http://www.bbc.co.uk/ genes

The BBC - characteristically on top of what makes its audience tick - has embraced the challenge of sating the public's thirst for stories on genetics by running a season of television and radio programmes on important issues in human genetics. For those who haven't yet had the benefit of tuning in you can turn to the interactive web site that accompanies the broadcasts, which contains accessible and attractively presented information on genetic topics across the spectrum - from the basic rules of inheritance to why we age, and genetic diseases, through to gene profiling and human reproduction.

The site guides you aboard the 'Gene Safari', where you can learn about the cloning process and about GM organisms; or catch up with the 'DNA detectives', who reveal that Adam and Eve could never have met; or enter a courtroom debate about whether you'd want to live forever. Presenting so much about genetics as accurately and engagingly as they have done can't be easy; however, some sections border on the trivial - that genetics will tell us about our love lives, for example - or could be a bit misleading.

This web site is better suited for browsing than for systematic learning. For those wishing to venture beyond the surface, however, useful resources and links are provided. A selling feature of this site is the live debates that take place for an hour after each broadcast, allowing visitors to quiz geneticists on the programme's featured topics. And for geneticists who want a deeper insight into what the public really thinks, the 'message board' offers some veritable gems.

Tanita Casci

\title{
Fission chips here we come!
}

The number of sequenced eukaryotic genomes has just increased from five to six. As reported in Nature, Schizosaccharomyces pombe, or fission yeast, is the second unicellular eukaryote to be sequenced - the first being its higher profile relative, Saccharomyces cerevisiae. The functional and comparative analysis of this new genome sequence will provide key insights into the evolution and function of eukaryotic cells.

The sequencing itself was carried out by the Wellcome Trust Sanger Institute in collaboration with several other laboratories, and the quality of the data is extremely high - less than 1 error per $10^{5}$ bases. As for completeness - a relative term in the context of genome sequencing - the $S$. pombe sequence rates highly too. All but $\sim 1.1 \mathrm{Mb}$ (mostly tandem repeats of ribosomal RNA genes) of the 13.8-Mb genome has been sequenced.
The genome has been annotated using a mixture of $a b$ initio gene prediction and sequence comparison, and from this analysis it is estimated that $S$. pombe has 4,824 genes, about 750 fewer than $S$. cerevisiae. Introns are present in $43 \%$ of genes and there are 4,730 of them in the genome, more than 20 times the number present in $S$. cerevisiae. The centromeres of the three chromosomes range in size from 35 to $110 \mathrm{~kb}$ and comprise arrays of tandem repeats, which makes them more similar than those of $S$. cerevisiae to the centromeres of larger eukaryotes. Interestingly, although proteincoding genes are absent from the centromeric sequences, there are quite a few tRNA genes, and, on the basis of the locations of these genes, they might delimit functional domains within the centromere.

In a comparison of the genomes of the two yeasts, the authors found that only $16 \%$ of the fission yeast genes do not have homologues in
S. cerevisiae. When this comparison included prokaryotic, as well as the other eukaryotic, genome sequences, a group of genes was found that is present only in eukaryotes. Such genes help to define the key molecules, mechanisms and functions that are unique to eukaryotic biology. The genes identified so far are involved in the cytoskeleton, intracellular compartmentalization, the ribosome and several other eukaryote-specific processes. The authors did a similar comparison to find genes that are specific to multicellular organisms, but few of these were identified. However, in an accompanying News and Views, Jonathan Eisen points out that this analysis did not take into account that multicellularity probably evolved independently in plants and animals. This question will therefore have to wait for more representative genomes to be completed.

The genome sequence of fission yeast reveals that there is a lot in common between $S$. cerevisiae and $S$. pombe. But there are some important differences as well - for example, in centromere structure and the amount of RNA splicing which means that $S$. pombe is the better experimental system for studying some aspects of eukaryotic

\section{EVOLUTION}

\section{Feel the fourth}

In the fast-moving field of Drosophila genetics and genomics (see the reviews on pages 176 and 189 of this issue), chromosome 4 , the smallest of the three autosomes, has often kept a rather low profile. Looking no bigger than a dot on metaphase spreads and with only $\sim 80$ genes to its name, not to mention the lack of crossing over during meiosis, most labs have shunned this dull and invariable chromosome. And with good reason, right? Wrong, say Wen Wang et al., who have just published work showing that the fruitfly's fourth chromosome leads a happening life: not only does it recombine, but also it consists of blocks of highly polymorphic regions interspersed with less variable domains. Both findings suggest that, contrary to theoretical and empirical evidence, this chromosome has been evolving, albeit at different rates in different regions.

Only $\sim 25 \%$ of chromosome 4 is euchromatic, and it is in this portion of the chromosome that Wang and colleagues decided to look for nucleotide variation, by sequencing a $5-\mathrm{kb}$ chromosome 4 region from 33 individuals taken from either worldwide or local (Israeli) fly populations. Surprisingly, in both samples, nucleotide variation was far in excess of that seen before on a chromosome 4 gene (cubitus interruptus, ci), but was comparable with that seen on autosomes. What's more, the segregating nucleotides are organized into two haplotypes. The fact that the dimorphism occurs at all and that the frequency of each haplotype is more or less equal in local and worldwide populations probably points to the influence of balancing selection - a force that actively maintains genetic variation. Intrigued by the difference in variation at $c i$ 


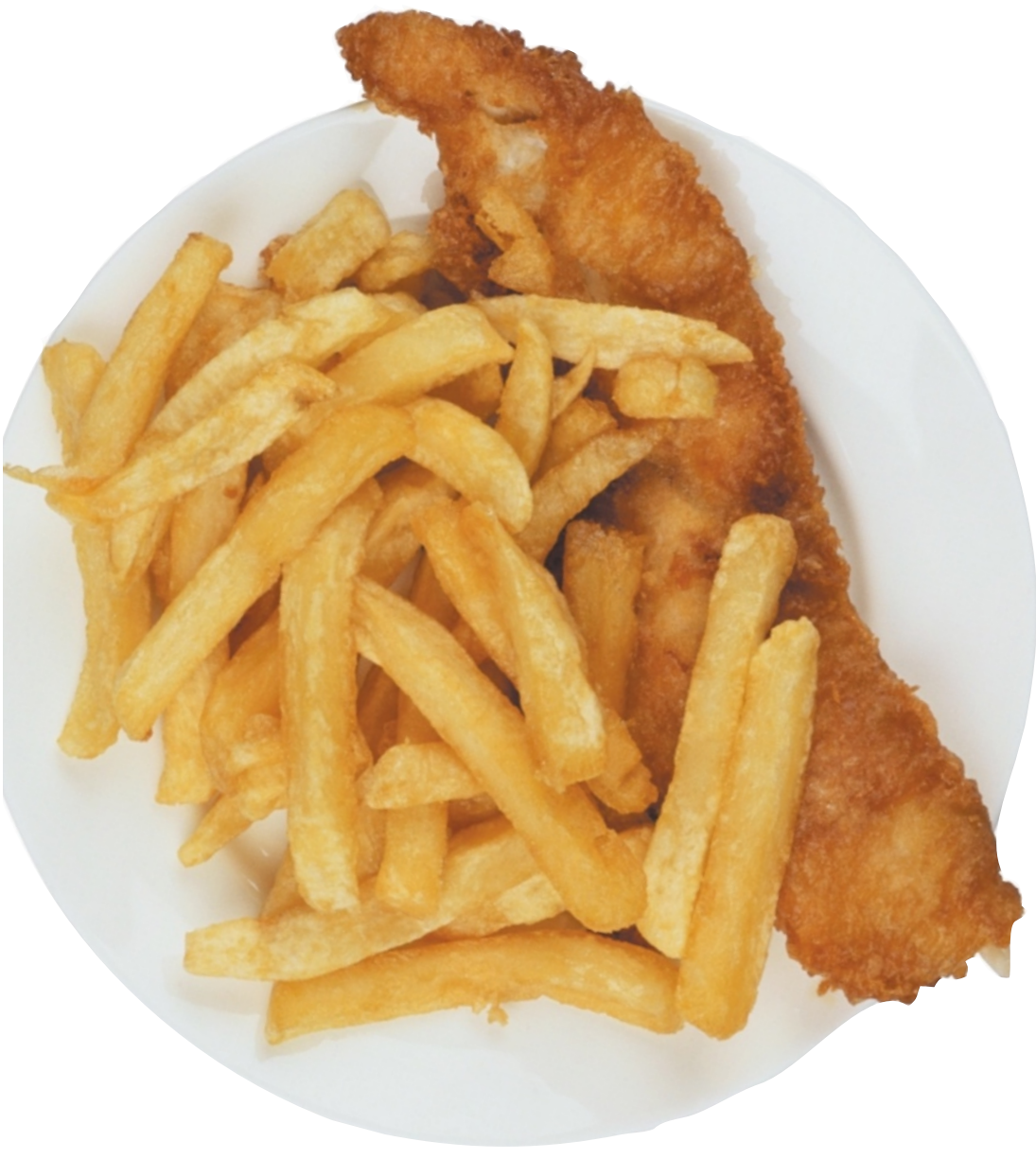

biology. The good news for $S$. pombe researchers is that their system is now officially postgenomic, and the study of this important little organism can now take advantage of an array of functional genomics approaches.

Mark Patterson

compared with their sample region, the authors sequenced an additional 15 regions. Their survey found that chromosome 4 is made up of three distinct domains: the region organized into haplotypes was flanked, on one side, by a variable region with no haplotype organization and, on the other, by a very homogeneous region.

What could account for the different evolutionary histories of the three domains? The authors estimate that at least six recombination events have occurred along the chromosome if recombination were taking place at the boundaries between different domains - which is suggested by the presence of repetitive sequences - then this could be a contributing factor.
6) References and links ORIGINAL RESEARCH PAPER Wood, V et al. The genome sequence of Schizosaccharomyces pombe. Nature 21 February 2002 (10.1038/nature 724) FURTHER READING Eisen, J Brouhaha over the "other yeast". Nature 21 February 2002 (10.1038/nature 725)

\section{WEB SITE}

The S. pombe Genome Sequencing Project: http://www sangerac.uk/Projects/S pombe

However, the specific forces that give each region its characteristic variability have yet to be discovered.

Whether this revised view of the fourth chromosome will be enough to bring it out from the shadows is debatable; after all, its recombination rate might be several hundred times lower than that of autosomes. Then again, it could be a hunting ground for some more surprises.

Tanita Casci

(4) References and links ORIGINAL RESEARCH PAPER Wang, W.

et al. Nucleotide variation along the Drosophila melanogaster fourth chromosome. Science 295, 134-137 (2002)

\section{WEB SITE}

Manyuan Long's lab: http://pondside. uchicago.edu/ceb/faculty/long.html
IN BRIEF

\section{BEHAVIOURAL GENETICS}

Loss of sex discrimination and male-male aggression in mice deficient for TRP2.

Stowers, L. et al. Science 31 January 2002 (10.1126/science1069259) $\operatorname{Trp} 2$ is exclusively expressed in neurons of the pheromone-sensing vomeronasal organ (VNO) in mice and encodes a putative ion channel of the transient receptor potential family. Stowers et al. now report that TRP2 is required for VNO neurons to be activated by urine-borne pheromones and that $\operatorname{Tr} p 2^{-/-}$male mice fail to behave aggressively towards other males, and display courtship and mounting behaviour to both sexes, so indicating that VNO function is required for sex discrimination and certain genderspecific behaviours.

\section{GENOMICS}

Short interspersed transposable elements (SINEs) are excluded from imprinted regions in the human genome.

Greally, J. M. Proc. Natl Acad. Sci USA 99, 327-332 (2002)

Imprinted regions are epigenetically modified, but do they have unique genomic characteristics? By comparing the nucleotide sequence and retro-element composition of imprinted and nonimprinted human loci, John Greally has found that maternally and paternally imprinted loci differ in $\mathrm{G} \cdot \mathrm{C}$ composition and in retro-element content. Additionally, SINEs seem to be excluded from imprinted regions, perhaps because they attract and spread methylation - a process that has to be tightly regulated at imprinted loci.

\section{GENETIC SCREENS}

fzr-1 and lin-35/Rb function redundantly to control cell proliferation in $C$. elegans as revealed by a nonbiased synthetic screen.

Fay, D. S. et al. Genes Dev. 16, 503-517 (2002)

When a gene knockout produces little phenotypic effect, it does not say much about a gene's function. But mutation in a second, interacting, gene can sometimes elicit a clearcut phenotype, so revealing the function of both genes. Fay et al. have devised an elegant screen for such 'synthetic' mutations in Caenorhabditis elegans, and have discovered new functions for the worm retinoblastoma gene homologue.

\section{HUMAN GENETICS}

\section{Mutant chromatin remodeling protein SMARCAL1} causes Schminke immuno-osseous dysplasia.

Boerkoel, C. F. et al. Nature Genet. 30, 215-220 (2002)

Its diverse symptoms and variable severity flag Schminke immuno-osseous dysplasia (SIOD) as a genetically heterogeneous disease. But now Boerkoel et al. report that mutations in SMARCAL1, which belongs to the SWI/SNF2 family of chromatin remodelling proteins, underlie SIOD. The authors also found genotype-phenotype correlations that might account for SIOD's clinical heterogeneity. How these mutations affect transcriptional activation to cause SIOD requires further investigation. 